\title{
INEQUALITIES AMONG EIGENVALUES OF DIFFERENT SELF-ADJOINT DISCRETE STURM-LIOUVILLE PROBLEMS
}

\author{
HAO ZHU AND YUMING SHI
}

\begin{abstract}
In this paper, inequalities among eigenvalues of different self-adjoint discrete SturmLiouville problems are established. For a fixed discrete Sturm-Liouville equation, inequalities among eigenvalues for different boundary conditions are given. For a fixed boundary condition, inequalities between the $n$-th eigenvalues for two different equations are given. These results are obtained by applying continuity and discontinuity of the $n$-th eigenvalue function, monotonicity in some direction of the $n$-th eigenvalue function, which were given in our previous papers, and natural loops in the space of boundary conditions. Some results generalize the relevant existing results about inequalities among eigenvalues of different Sturm-Liouville problems.
\end{abstract}

Mathematics subject classification (2010): 39A70, 34B24, 15A42, 47A75.

Keywords and phrases: Inequality, discrete Sturm-Liouville problem, eigenvalue, self-adjointness.

\section{REFERENCES}

[1] F. V. AtKinson, Discrete and Continuous Boundary Problems, Academic Press, New York, 1964.

[2] M. Bohner And O. Dosly, The discrete Prüfer transformation, Proc. Amer. Math. Soc. 129, 9 (2001), 2715-2725.

[3] X. CaO, Q. Kong, H. Wu And A. Zettl, Sturm-Liouville problems whose leading coefficient function changes sign, Canadian J. Math. 55, 4 (2003), 724-749.

[4] E. A. Coddington And N. Levinson, Theory of Ordinary Differential Equations, McGraw-Hill, New York, 1955.

[5] R. Courant And D. Hilbert, Methods of Mathematical Physics, Interscience Publishers, New York, 1953.

[6] M. Eastham, Q. Kong, H. Wu And A. ZetTL, Inequalities among eigenvalues of Sturm-Liouville problems, J. Inequal. Appl. 3, 1 (1999), 25-43.

[7] A. JiRARI, Second-order Sturm-Liouville difference equations and orthogonal polynomials, Mem. Amer. Math. Soc. 113, 542 (1995).

[8] Q. Kong, Q. Lin, H. WU ANd A. Zettl, A new proof of the inequalities among Sturm-Liouville eigenvalues, PanAmerican Math. J. 10, 2 (2000), 1-11.

[9] Q. Kong, H. Wu AND A. Zettr, Dependence of the $n$-th Sturm-Liouville eigenvalue on the problem, J. Differential Equations 156, 2 (1999), 328-354.

[10] Q. Kong, H. WU AND A. ZETTL, Inequalities among eigenvalues of singular Sturm-Liouville problems, Dynamic Systems \& Appl. 8, 3-4 (1999), 517-531.

[11] Q. Kong, H. Wu AND A. Zettl, Geometric aspects of Sturm-Liouville problems, I. Structures on spaces of boundary conditions, Proc. Roy. Soc. Edinb. Sect. A Math. 130, 3 (2000), 561-589.

[12] Q. Kong, H. Wu And A. ZetTl, Left-definite Sturm-Liouville problems, J. Differential Equations 177, 1 (2001), 1-26.

[13] Q. Kong, H. Wu And A. Zettl, Sturm-Liouville problems with finite spectrum, J. Math. Anal. Appl. 263, 2 (2001), 748-762.

[14] Q. Kong, H. Wu And A. Zettl, Singular left-definite Sturm-Liouville problems, J. Differential Equations 206, 1 (2004), 1-29. 
[15] W. Peng, M. Racovitan And H. Wu, Geometric aspects of Sturm-Liouville problems, V. Natural loops of boundary conditions for monotonicity of eigenvalues and their applications, Pac. J. Appl. Math. 4, 4 (2006), 253-273.

[16] Y. SHI AND S. CHEN, Spectral theory of second-order vector difference equations, J. Math. Anal. Appl. 239, 2 (1999), 195-212.

[17] Y. SHI AND S. CHEN, Spectral theory of higher-order discrete vector Sturm-Liouville problems, Linear Algebra Appl. 323, 1-3 (2001), 7-36.

[18] H. SUN AND Y. SHI, Eigenvalues of second-order difference equations with coupled boundary conditions, Linear Algebra Appl. 414, 1 (2006), 361-372.

[19] Y. WANG AND Y. SHI, Eigenvalues of second-order difference equations with periodic and antiperiodic boundary conditions, J. Math. Anal. Appl. 309, 1 (2005), 56-69.

[20] J. Weidmann, Spectral Theory of Ordinary Differential Operators, Lecture Notes in Mathematics, vol. 1258, Springer-Verlag, Berlin, 1987.

[21] A. Zettl, Sturm-Liouville Theory, Mathematical Surveys Monographs, vol. 121, Amer. Math. Soc., 2005.

[22] H. ZHU AND Y. SHI, Continuous dependence of the $n$-th eigenvalue of self-adjoint discrete SturmLiouville problems on the problem, J. Differential Equations 260, 7 (2016), 5987-6016.

[23] H. Zhu, S. Sun, Y. SHI AND H. Wu, Dependence of eigenvalues of certain closely discrete SturmLiouville problems, Complex Anal. Oper. Theory 10, 4 (2016), 667-702. 